\title{
ELEKTRON LUG'ATLAR, TEZAURUS, ONTOLOGIYALAR ELEKTRON TA'LIMDA ZARURIY VOSITALAR SIFATIDA
}

Botir Elov

Alisher Navoiy nomidagi Toshkent davlat

o'zbek tili va adabiyoti universiteti Axborot texnologiyalari kafedrasi mudiri, t.f.n $\mathrm{PhD}$

Umid Yodgorov

Alisher Navoiy nomidagi Toshkent davlat o'zbek tili va adabiyoti universiteti, Kompyuter lingvistikasi mutaxassisligi 1-bosqich magistranti

Annotatsiya. Hozirda deyarli barcha sohaning elektron nashrlari mavjud. Elektron ta'lim vositalari allaqachon tizimga keng joriy etilib, dars jarayonida samarali foydalanilmoqda. Ushbu maqola elektron darsliklar, lug'atlar, tezauris va boshqa manbalar yaratish to'g'risida fikr yuritadi.

Kalit so'zlar: elektron lug'at, tezauris, axborot, dastur, dasturchi.

Annotation. Electronic publications are now available in almost all industries. E-learning tools are already widely introduced into the system and are used effectively in the teaching process. This article focuses on creating etextbooks, dictionaries, thesauruses, and other resources.

Keywords: electronic dictionary, thesaurus, information, program, programmer.

Elektron ta'lim resurslarini yaratish jarayoniga turli yo'nalish bo yicha mutaxasislarni jalb qilish zarur, hech bo `lmaganda ularning fikrlarini o`rganib chiqish kerak. Bunday mutaxasislar qatoriga quyidagilar kiradi:

-o`qituvchi;

-psixolog;

- testolog- o`qitish natijalarini nazorat qilish usullari bo`yicha

-mutaxasis;

- shakllar dizayneri yoki web usta;

- dasturchi.

Elektron o`quv resurslarini yaratish jarayonida psixologik-pedagogik, texniktexnologik, estetik va ergonomik talablar qo`yiladi. Elektron darslik, Elektron 
ma'lumotnoma va uslubiy qo'llanma kabi dasturiy mahsulotlar qo`yilgan didaktik talablarga javob berishi kerak. Didaktik talablar ta'lim berishning spetsifik qonuniyatlariga va mos ravishda ta'lim berishning didaktik tamoyillariga mos kelishi kerak.

Dasturni yaratish jarayoni aniq mantiqiy davom etadigan harakatlardan iborat bo lib, ularni bajarish natijasida ishga yaroqli, qonun-qoidalarga asosan rasmiylashtirilgan dastur mahsuloti yaratiladi. Dastur buyurtmachining texnik shartlariga javob berganda ishga yaroqli deb hisoblash mumkin. Uni o`quv jarayonida qo`llash yoki Internet tarmog`iga ulangan kompyuterga joylashtirish mumkin.

Axborot asrida insoniyat tarixida sanoat va fan olamida olamshumul yutuqlar qo'lga kiritildi. Dunyoda axborot eng qimmat narsaga aylandi. Kompyuter ixtiro qilinishi insonlar bajaradigan yumushlarni yengillashishiga olib keldi. Fan, ta'lim sohalarida o'qitish o'rganishning zamonaviy vositalari joriy qilindi.

Ta'lim tizimini uslubiy va dasturiy ta'minotiga yo'naltirilgan ilmiy tadqiqot ishlarida o'quv jarayonini tashkil etish o'tkazish va boshqarishni avtomatlashtirishda elektron ta'lim resurslari markazini tashkil etish muhim ahamiyat kasb etishi ta'kidlanadi

A. P. Tixomirov, V. I. Soldatkinlarni fikriga ko'ra chet mamlakatlar ta'lim tizimida kechayotgan jarayonlar tahlili shuni ko'rsatdiki, o'qitish tizimi evolyutsion xarakterga ega bo'lib, masofaviy yoki kompyuterli o'qitish texnologiyasi klassik ta'limdan virtual ta'limga o'tadi

Shuningdek, tadqiqotlarda o'quv jarayoniga informatsion birinchi navbatda masofaviy o'qitish texnologiyalarini qo'llash ta'lim samaradorligini sezilarli darajada oshirishga, o'qitish vaqtini qisqartirishga erishish mumkinligi asoslab beriladi.

Zamonaviy axborot texnologiyalarining rivojlanishi ta'lim jarayonini masofadan turib tashkil etish, boshqarish, nazorat qilish imkoniyatlarini oshiradi. Natijada masofali ta'lim kompyuterli ta'limning eng etakchi maqomini ola boshladi

1. Elektron o'quv nashrlari - o'quv dasturiga mos keluvchi turli ma'ruzalar, seminarlar, laboratoriya ishlari va keys texnologiyalar.

2. Multimediali o'rgatuvchi dasturlar - animatsiya va tovushli kuzatuvli o'quv kurslari.

3. Elektron darsliklar - o'qitishni avtomatlashtirishga mo'ljallangan 
elektron o'quv nashri.

4. Elektron o'quv qo'llanmalar - fanning alohida bo'limlari, mashqlar yoki masalalar to'plami, ma'lumotnoma va boshqalar. D. Sayfurov fikricha elektron o'quv adabiyotlari an'anaviy o'qitish shakllari kamchiliklarini bartaraf etish imkonini beradi Keyingi yillarda elektron o'quv adabiyotlarini yaratish va elektron lug'atli- ma'lumotli materiallarni ishlab chiqish keskin rivojlandi. Elektron o'quv nashrlarni tarmoq orqali tarqatish imkoniyati yaratildi. Elektron o'quv adabiyotlarining yana bir ahamiyatli jihati shundaki, undagi o'quv materiali, tasviriy materiallar eskirmaydi va emirilmaydi, saqlash uchun ko'p maydon va hajmni talab etmaydi. Elektron o'quv adabiyotlarni yaratish bosma nashrlardan ko'ra sezilarli darajada oshib bormoqda.

Ta'limda qo'llaniladigan elektron o'quv vositalari turlari va tavsifi. Hot Potatoes, iSpring dasturlari va uning imkoniyatlari. iSpring Suite asbob uskunalari orqali elektron darslik, videoma'ruzalar, elektron nazorat testlari, so'rovnomalar, tarmoqlangan dialogli elektron kurslarni va onlayn-perezentatsiyalarni yaratish. Prezi onlayn-perezentatsiyalarni yaratish dasturi. CoursLab dasturi va uning imkoniyatlari. Mytest - test yaratish va o'tqazish dasturlari.

Elektron axborot ta'lim resurslarini yaratishda keng ishlatilib kelinayotgan dasturiy ta'minotlardan biri Ispring dasturi hisoblanadi. Odatda, taqdimotni o'tkazishga tayyorlanish jarayonida aksariyat hollarda Microsoft PowerPoint dasturiy ta'minotidan foydalaniladi. Ammo bunday taqdimotlar faqat mazkur mahsulot formatidagina bo'lishi mumkin (ppt, pptx). Hozirgi vaqtda internet texnologiyalarining rivojlanishi va o'z navbatida, masofali ta'lim turining paydo bo'lishi natijasida taqdimot fayllarini internet brauzerining o'zida onlayn ravishda to'g'ridan-to'g'ri ko'rish uchun flash (swf) formatida yoki HTML 5 texnologiyasi asosida yaratilgan fayl bo'lishi kerak. Hozirga kelib, PowerPoint dasturida tayyorlangan taqdimotdan flash-rolik shakllantirish imkoniyatini beruvchi dasturlar yaratilgan.

Mahsulot iSpring deb nomlanadi va iSpring Free, iSpring PRO va iSpring Presenter kabi variantlarga ega. Mustaqil ekspertlarning fikriga ko'ra, bugungi kunda mazkur mahsulot tezligi, bir formatdan boshqa formatga konvertatsiyalash sifati va optsiyalar soniga ko'ra eng yaxshilaridan biri hisoblanadi. iSpring nafaqat flash-taqdimotlarni yaratishga, balki ta'lim jarayonida qo'llanilishi mumkin bo'lgan roliklar tayyorlashda, xususan, ularga turli shakldagi so'rovlar, elektron testlarni ham kiritgan holda o'zaro interaktiv bog'lanish imkoniyatini ham beradi.

iSpring quyidagi imkoniyatlari mavjud: 
- taqdimot fayllarini bir necha (exe, swf, html) formatlarda konvertatsiyalash imkoniyati;

- taqdimot kontentiga tashqi resurslarni (audio, video yoki flash fayllarni) kiritish imkoniyati;

- taqdimot kontentini muhofaza qilish: parol yordamida ko'ra olish, taqdimotga «himoya belgi»si qo'yish, taqdimotni faqat ruxsat etilgan domenlardagina «aylantirilishi»;

- video qo'shish va uni animatsiyalar bilan sinxronlashtirish;

- elektron test(nazorat)larini yaratish va natijalarini elektron pochtaga yoki masofaviy o'qitish tizimiga (LMS) uzatib berish imkoniyatini beradigan interaktiv matnlar yaratish uchun vosita o'rnatilgan (Quiz tugmachasi);

- masofaviy o'qitish tizimida foydalanish uchun SCORMG'AICC $-\operatorname{mos}$ keluvchi kurslarini yaratish;

- taqdimot dastur darajasida aylantirish uchun ActionScript API;

videotasvirni yozish va uni taqdimot bilan sinxronlashtirish;

- YouTube'ga joylashtirilgan roliklarni taqdimot tarkibiga kiritish imkoniyati.

Ayni paytda yangi axborot texnologiyalari sohasida gipermedia tizimlarini qo'llash rivojlanib bormoqda. Bunday texnologiyalar asosida an'anaviy o'quv matnini yanada takomillashtirilgan o'quv materiali asosida kengaytirish va chuqurlashtirish hamda kurslar va animatsion lavhalardan foydalanish yo'li bilan almashtirish g'oyasi yotadi. Bunda u yoki bu holda ajratib berilgan matn lavhalari orasida o'zaro bog'anish tugunlari barpo etiladi. Mutaxassislarning ta'rifiga ko'ra, gipermatn inson intellektining katta xajmdagi axborotni esda saqlash kobiliyatini va mazkur axborotlar ichidan kommunikatsiya (muloqot) va tafakkur jarayonlarini assotsiatsiyalash yo'li bilan qidiruv ishlarini olib borishni imitatsiya (o'zida aks) qiladi. Boshqacha qilib aytganda, gipermatn murakkab darajada tashkil etilgan o'quv materiallari tizimi bo'lib, ko'plab statistik va dinamik axborotlarni o'zida mujassamlashtiradi hamda umumlashgan tarmoq tuzilishiga ega bo'ladi. Bunda axborot lavhalari ahamiyatini matn, grafik, sxema, videolavha, ijrochi dastur va animatsiya (qarakatli jarayon)lar o'ynaydi. Matnlar esa, o'z navbatida, yanada kichik matnchalardan tashkil topib, ular «matryoshka» («qo'g'irchoq ichida qo'g'irchoq») ko'g'irchog'i kabi ko'p marta ichma-ich joylashishlari mumkin. Bir matndan ikkinchisiga o'tish (chiqarish) EDning tarkibiga kiruvchi ma'lum munosabat orqali amalga oshiriladi. Matnlar orasidagi o'zaro bog'lanishlardan tashqari matn va videolavhalar, matn va ijrochi dastur hamda matn va animatsion effektlar orasida ham bog'lanishlar mavjud bo'lishi zarur. Bu bog'lanishlar ham 
ma'lum nisbatlar to'plamida keltirilgan nisbatlar ko'rinishida berilgan bo'ladi. Biz gilermatnlarni graf-daraxt ko'rinishida tasvirlashimiz mumkin, bunda matnlarning lavhalari, grafik tasvirlar, videolavhalar, ijrochi dasturlar va animatsiyalar doirachalar (graf tugunlari) ko'rinishida, ular asosidagi munosabatlar esa, mos doirachalarni tutashtiruvchi yoylar shaklida ifodalanadi. Shuni alohida qayd etish lozimki, gipermatnlardan foydalanishning samaradorligi ko'p jixatdan bog'lanishi mumkin bo'lgan axborotlarning uslubiy nuqtai nazardan maqsadga muvofiqligiga bog'iq bo'ladi. Chunonchi, nisbatlar to'plami har bir elementga hos bo'lgan aniq vazifalarning muayyanlashtirilishi, ularning muqobillik darajalari bilan harakterlanadi. Matnlar lavhalarida izox talab etuvchi («kalit») so’zlar, tushunchalar, matnning boshqa parchalari, jumladan, videolavhalar bilan bog' langanligini ko'rsatish maqsadida alohida rang bilan ajratilgan (yoxud tagiga chizilgan) holda berilishi mumkin. Shunday qilib, gipermatn tizimidan foydalanuvchilar graf tugunlari bo'ylab «sayohat»ga chiqib, uning uchlaridan mos axborot bo'lagini, yoylaridan esa, foydalanish tartibini aniqlashlari mumkin. Foydalanuvchining axborot lavhalari bo'ylab bunday «sayohati» navigatsiya deyiladi. Gipermatn tizimi, kitobni varaqlagan kabi yoxud kitobning mundarijasi bo'yicha (boblar, paragraflar va betlarni) ierarxik kuzatish singari, matnlarni ketma-ket qarab chiqish, shuningdek, oldindan ma'lum bog'lanish «yo'llari» bo'yicha ixtiyoriy yo'nalishda navigatsiya qilish imkonlarini beradi .

Gipermatn hujjatlarini ishlab chiqishda ushbu instrumental vositalar: Microsoft Front-Page (HTML-Hyper Text Markup Language), Alliare Home Site (HTML), Microsoft Power Point, Microsoft Word va boshqalardan foydalaniladi. Strategik illyustratsion o'quv materiallarini (turli manzaralar)ni yaratishda rastorli yoki vektorli rasmlar bilan ishlovchi dasturlardan foydalanish zarur bo'ladi. Ularga Corel Draw, Corel Xara, Corel Photo Paint, Adobe Photo Shop, Adobe Illustrator va boshqalar kiradi. Dinamik illyustratsion o'quv materiallari roliklarini yaratishda esa, ularni tuzish uchun maxsus muharrirlar va quyidagi Web-animatorlardan foydalaniladi: Disreet 3D Studio MAX, Alais Wave Front, Maya, Light Wave, Soft Image 3d, Adobe Image Ready, Gif Animator, Macromedia Flash, Adobe Premier va boshqalardan foydalaniladi. Tovush bilan kechadigan yozuvlar va tovushni taxrir qilish Sonic Foundry Sound Forge, Wave Lab, Sound Recorder va boshqa dasturlar yordamida amalga oshiriladi. 


\section{Foydalanilgan adabiyotlar:}

1 A.K.Ibragimov. Catalog of training tasks for training special endurance of yong gril handboll players // Academical.An International Multidisciplenary Research Journal 2.35-39p

2. A.K.Ibragimov, F.I.Mo'minov. Methodology of optimization of trainingfor young players // International Journal For Innovative Engineering and Management Research Vol 10 Issue02, Feb2021 ISSN 2456 - 5083 Page 43-48.

3 R.R.Raxmonov. Distribution of training loads at the stage of competitive preparation for middle runners // ACADEMICIA: An International Multidisciplinary Research Journal https://saarj.com ISSN: 2249-7137 Vol. 11, Issue 2, February 2021 Impact Factor: SJIF $2021=7.492$ Pages 768-777. 